\title{
NOTE ON NIELSEN TRANSFORMATIONS ${ }^{1}$
}

\section{ELVIRA STRASSER RAPAPORT}

Let $a_{1}, \cdots, a_{n}$ be free generators of the free group $F_{n}, c_{1}, \cdots, c_{n}$ those of the free group $F_{n}{ }^{\prime}, N$ an isomorphism of $F_{n}$ onto $F_{n}^{\prime}$, and $b_{i}=N\left(a_{i}\right)$ the maps of the $a_{i}$ under $N$. Then the $b_{i}$ are generators not only of $F_{n}^{\prime}$ but of any quotient group $G^{\prime}$. I shall say that the generators $b_{i}$ of $G^{\prime}$ arise from the $a_{i}$ by a Nielsen transformation, $N$. In case $F_{n}=F_{n}^{\prime}, N$ is (Nielsen) automorphism of $F_{n}$ but need not be an automorphism of $G=G^{\prime}$; nor is every automorphism of $G$ expressible in terms of some $N$ acting on a given set of generators. However, a given automorphism of $G$ can be written as a Nielsen transformation acting on a suitably large set of generators (Theorem 2). I shall discuss this and related matters in the present paper.

The following definitions and notation will be used.

$G=\left\{a_{1}, \cdots, a_{n} ; R_{1}, \cdots, R_{k}\right\}=\{a ; R\}$ will denote the group given on $n$ generators $a_{i}$ and $k$ generating relations

$$
R_{j}\left(R_{j}=R_{j}\left(a_{1}, \cdots, a_{n}\right)=1 .\right.
$$

$G^{\prime}$ will denote the commutator subgroup of $G$.

$\bar{x}$ will denote the inverse of $x$.

$[x, y]=x y \bar{x} \bar{y}$.

$I \sim N$ will denote the following. If $G=\{a ; R\} \simeq I G=\{b ; S\}$ under the mapping $I\left(a_{i}\right)=v_{i}(b)$ and there exist relations $s_{i}^{\prime}(b)$ in $I G$ for which the elements $v_{i}(b) s_{i}^{\prime}(b)$ generate $b$ freely, then the isomorphism $I^{\prime}$ given by $I^{\prime}\left(a_{i}\right)=v_{i}(b) s_{i}^{\prime}(b)$ is a Nielsen transformation, $N$. I shall say that $I$ is equivalent to $N: I \sim N$. Similarly for automorphisms, $A$, that is in case $I G=G$.

The group $K=\left\{a ; a^{p}\right\}$ has, for $a=a_{1}$ and prime $p$, automorphisms $A$, given by $A(a)=v(a)$, for which $v(a)$ does not generate $F_{1}(a)$. For example, if $p=3, A(a)=a^{2}$ is such an automorphism. However, $a^{2}=\bar{a}$ in $K$, so that the automorphism given by $A^{\prime}(a)=\bar{a}=a^{2} \bar{a}^{3}=a R^{\prime}$, a Nielsen transformation, of $K$, is equivalent to $A$. For $p>3$ and prime $q<p, A(a)=a^{q}$ is an automorphism of $K$, but $A \nsim N$.

There exist other groups with one defining relation having nonNielsen automorphisms:

THEOREM 1. The group $L=\{u, v ; u$ ivī̄v̄ $\bar{u} v u\}$ has an automorphism

Received by the editors July 11, 1957.

1 This work was stimulated by the group theory seminar held at New York University, 1956-1957, sponsored by the National Science Foundation. 
$A, A(u)=\bar{v} \bar{u} v u, A(v)=\bar{u} \bar{v} \bar{u} A(\bar{u})$, which is not equivalent to a Nielsen transformation.

$L$ is the group of Listing's knot, the automorphisms of which were first studied by Dehn [6].

Proof. Suppose $A \sim N$. The commutator subgroup $L^{\prime}$ contains $u$ (since the generating relation $R$ implies $\bar{u}=[u v, \bar{u} \bar{v}]$ ), hence it contains all conjugates $v^{k} u \bar{v}^{k}$; moreover, $L^{\prime}$ is a free group generated by $u$ and $v u \bar{v}=y[1]$. The factor group $L / L^{\prime}=F(v)$ is also given by $L / C$, where $C$ is the normal subgroup generated by $u$, and $A$ induces an automorphism of $L / C=\{u, v ; u\}$. Since every Nielsen transformation of a group $G=\left\{a ; R_{1}\right\}$ maps the defining relation $R_{1}$ onto a conjugate of the defining relation, or of its inverse, of the image group $K=\left\{b ; S_{1}\right\}$ [1], the assumption $A \sim N$ implies that an automorphism $N$, equivalent to $A$, of $L$ is given by $N(u)=x u^{\epsilon} \bar{x}, x \subset L, \epsilon= \pm 1$, hence that $A(u)=\bar{v} \bar{u} v u=x u^{\epsilon} \bar{x}$ in $L$, and $A^{2}(u)=\bar{u}=A(x) A\left(u^{\epsilon}\right) A(\bar{x})$ is a conjugate $w u \bar{w}$ of $u$ for some element $w$ in $L$. This implies $w u \bar{w} u=1$ in $L$, which will prove false.

If $w=1$ in $L, \bar{u}=u$ follows, which does not hold in the free group $L^{\prime}(u, y)$; since $w u \bar{w} u$ is not the empty word, $w$ is not in $L^{\prime}$ (otherwise $L^{\prime}(u, y)$ would contain a nontrivial relation: $w u \bar{w}=f(u, y)$ $=g(u, y) u \bar{g}(u, y)$ would equal $\bar{u})$.

Let $f=f(u, y), g=g(u, y)$ be elements in $L^{\prime}$. Since every element of $L$ has a unique representation of the form $v^{h} f, w=v^{k} f$ with $k \neq 0$. This gives $w u \bar{v}^{2}=v^{k} f u \bar{f} \bar{v}^{k}$; setting $f u \bar{f}=g$, wuw $=v^{k} g \bar{v}^{k}$. If now $w u w \bar{v} u$ is a relation in $L$, then $v^{k} g \bar{v}^{k}=\bar{u}$, and $g(u, y)=\bar{v}^{k} \bar{u} v^{k}$. Again, since $u$ and $y$ generate $L^{\prime}$ freely, no relation of $L$ is a word in $u$ and $y$ alone, and so for any element $a$ in $L^{\prime}, a=h(u, y)$ uniquely; the above stated equality implies that for some integer $k \neq 0$ a conjugate of $u$ by $v^{k}$ is the $u, y$-word $\bar{g}=f \bar{u} \bar{f}$.

To see that no such $k$ exists, observe that $L$ is extension of $F_{2}(u, y)$ by $F_{1}(v)$, given by

$$
\begin{aligned}
L=\{v, u, y ; u u v \bar{u} \bar{v} \bar{u} v u, \bar{y} v u \bar{v}\} & =\left\{v, u, y ; v u \bar{v}=y, v y \bar{v}=\bar{u} y^{3}\right\} \\
& =\left\{v, u, y ; B(u)=y, B(y)=\bar{u} y^{3}\right\}
\end{aligned}
$$

where $B$ is the automorphism of $F_{2}(u, y)$ induced by conjugation with v. Thus, $v^{k} u \bar{v}^{k}=B^{k}(u), \bar{v}^{k} u v^{k}=B^{-k}(u)$.

The automorphism $B$ is characterized [5] by the matrix

$$
M=\left(\begin{array}{rr}
0 & 1 \\
-1 & 3
\end{array}\right)
$$

no power of which has eigenvalue -1 . It follows that $v^{k} u \bar{v}^{k}$ cannot be 
equal to $f \bar{u} \bar{f}$ for any $k$. Thus, $A$ is not equivalent to a Nielsen transformation. With this the theorem is proved.

Though the automorphism $A$, given by $A(v)=\bar{u} \bar{v} \bar{u} A(\bar{u}), A(u)$ $=\bar{v} \bar{u} v u$, is not equivalent to a Nielsen transformation in $L=\{v, u ; R\}$, $L$ can be given various presentations in which $A=N$. An example with 5 generators is given in [2]. The fewest possible number of generators to give $A=N$ is 3 ; the presentation mentioned in the proof above gives $A(u)=y \bar{u}^{2}, A(y)=\bar{u} y \bar{u}^{2} y \bar{u}^{2}, A(v)=\bar{v} \bar{y} u \bar{y}$.

These two examples are specific to the group $L$; the following one leads to a general procedure.

$$
L=\{u, v, x, y ; \bar{u}=[u v, \bar{u} \bar{v}], x=\bar{v} \bar{u} v u, y=\bar{u} \bar{v} \bar{u} \bar{x}\}
$$

yields $A(u)=x, A(x)=\bar{u}, A(v)=y, A(y)=u^{2} v u$, with $A=N$. In general, given any group $G$ and an automorphism $A$ of $G$, introducing $A^{k}(g)$ as new generators $g^{*}(k)$ for every power $k$ of $A$ (in case $A$ has finite order, $h,|k|<h>0$ ) and every generator $g$, and adding the new relations $g^{*}(k) A^{k}(\bar{g})$, renders $A=N$. The following method is always finite for finitely generated $G$.

Theorem 2. Let $G=\{g ; R\}, A$ an automorphism of $G, A(g)=v(g)$; define $w(g)$ by $A(w(g))=g$; set $H=\{g, h ; R, \bar{h} w(g)\}, A^{\prime}(g)=h w(g) v(g)$, $A^{\prime}(h)=g$. Then $H=G, A^{\prime} \sim A$, and $A^{\prime}=N$.

In other words, every automorphism of a group can be effected by a Nielsen transformation acting on double the number of generators.

Proof. By the theorem of Tietze [3], $H=G$. Since $A^{\prime}(g)=v(g) \bmod$ relations in $H, A^{\prime}(w(g))=A^{\prime}(h)=g \bmod$ relations in $H, A^{\prime} \sim A$. Since $g$ is an image under $A^{\prime}$, all $g$-words are in the image group; then, because $A^{\prime}(g)=h \bar{w}(g) v(g)$, all $h$-words are in the image group; hence $A^{\prime}=N$.

The same can be accomplished for a given isomorphism $I$ of two groups.

Theorem 3. Let $G=\{g ; R\} \simeq I G=I I=\{h ; S\}, I(g)=v(h)$. Define $w(g)$ by $I(w(g))=w(v(h))=h$, and set $G^{*}=\left\{g, g^{*} ; R, g^{*} \bar{w}(g)\right\}, I I^{*}$ $=\left\{h, h^{*} ; S, h^{*} \bar{v}(h)\right\}, I^{*}(g)=h^{*}, I^{*}\left(g^{*}\right)=h$. Then $G^{*}=G, H^{*}=H$, $I^{*} \sim I$, and $I^{*}=N$.

In the case of the group $L$, there is a presentation for which: 1 . every automorphism, as well as 2. every isomorphism (to a group on the same number of relations) is a Nielsen transformation, namely $\{v, u, y ; R, \bar{y} v u \bar{v}\}$ above. 1 . The automorphism group of $L$, modulo inner automorphisms, is generated by $A$ given above, and $P$ given by $P(v)=\bar{v}, P(u)=\bar{u}[2]$. In this presentation, $A=N_{1}$ and as $P(y)$ 
$=P(v u \bar{v})=\bar{v} \bar{u} v=y \bar{u}^{3}$ in $L, P=N_{2}$. 2. If $I L=H=\left\{g, a, b ; S_{1}, S_{2}\right\}$, then $H / H^{\prime}=F_{1}$ and (up to a Nielsen transformation on $H$ ) $H / H^{\prime}=F_{1}(g)$, so that $H^{\prime}=F_{2}(a, b)$; hence $I(u)$ and $I(y)$ are automorphic images $A(a)$ and $A(b)$ of $a$ and $b$ in $F_{2}(a, b)$. Since $H / H^{\prime}=F_{1}(g)=I\left(L / L^{\prime}\right)$ $=I\left(F_{1}(v)\right)$, the image $I(v)$ must be of the form $g f f(a, b)$ modulo relations of $H$, where $\epsilon= \pm 1$. The mapping $I(v)=g^{\epsilon} f(a, b), I(u)=A(a)$, $I(y)=A(b)$ is clearly a Nielsen transformation.

The rest of the discussion concerns extensions of the free group on two generators, $F_{2}$, by the free cyclic group, $F_{1}$, in case the commutator subgroup of the extension is isomorphic to $F_{2}$.

Theorem 4. If $G=\{g, a ; S\} \simeq H=\{x, y ; R\}, S=S_{1}, R=R_{1}$ with $H / H^{\prime} \simeq F_{1}, H^{\prime} \simeq F_{2}$, then $G$ is Nielsen image of one of three nonisomorphic groups.

The proof is based on two lemmas; the verification of the first one is due to Magnus. ${ }^{2}$

Lemma 1. Set $a_{i}=g^{i} a \bar{g}^{i}, i: 0, \pm 1, \cdots ; \epsilon= \pm 1, r, k$ integers. The relation $S$ of Theorem 4 is (a conjugate of $S^{* \pm 1}=$ ) $a_{1}^{r} a_{2} a_{1}^{k} a_{0}^{\epsilon}$.

Proof. By considering the factor group $G / G^{\prime}$, it can be verified that for some Nielsen transform $N G, N G^{\prime} \supset a$; thus $a \subset G^{\prime}$ may be assumed. Then $G / G^{\prime}=F_{1}(g), G^{\prime}$ is generated by the $a_{i}$, and the sum of exponents of $g$ in $S$ is zero. A conjugate $g^{h} S \bar{g}^{h}=f\left(a_{0}, \cdots, a_{r+h=L}\right)$ generates the normal subgroup $\{S\} \subset F_{2}(g, a)$, so that one may assume $S=S\left(a_{0}, \cdots, a_{L}\right)$, with $a_{0}$ and $a_{L}$ actually occurring in $S$.

Define $K_{\imath}$ as that subgroup of $G^{\prime}$ generated by the elements $a_{0}, a_{1}, \cdots, a_{L}, \cdots, a_{t}$. Then $K_{L}$ is a free subgroup of $G^{\prime}$ having $L+1$ generators and the one relation $S\left(a_{0}, \cdots, a_{L}\right)=S_{L}$. Since $S_{L}$ is not the empty word, so that $K_{L}$ is not isomorphic to the free group on $L+1$ generators, its rank is at most $L$. Since the Betti number of $K_{L}$ abelianized is $L$, the rank is exactly $L$. Similarly for every $K_{t}$; thus $K_{t} \simeq F_{L}$. This shows: the ascending chain

$$
K_{L} \subseteq K_{L+1} \subseteq \cdots
$$

consists of subgroups of rank $L$ of $G^{\prime}$, with $K_{\infty}=G^{\prime}=F_{2}$; therefore [4], there is a number $h$ for which $K_{L+h}=G^{\prime}=F_{2}$, giving $L=2$, and so $S=S\left(a_{0}, a_{1}, a_{2}\right), K_{L+h}=F\left(B_{1}, B_{2}\right)=F_{2}(B), B_{1}, B_{2} \subset G^{\prime}$.

Set $S_{L+h+1}=U, a_{L+h+1}=u$; form the subgroup $D=\left\{K_{L+h}, u ; U\right\}$ of $G$. Then $D=G^{\prime}$. Since $u$ is an element of the free group $K_{L+h}=F(B)$, $u$ has a representation as a unique $B$-word, $u=f(B)$; then $D$ is given

2 This theorem is of some interest with respect to a conjecture of Magnus that if $G=\left\{a_{1}, \cdots, a_{n} ; R_{1}\right\} \simeq H=\left\{b_{1}, \cdots, b_{n} ; S_{1}\right\}$, then $H=N G$. 
by $\left\{B_{1}, B_{2}, u ; \bar{u} f(B)\right\}$ as well as by $\left\{B_{1}, B_{2}, a_{j}, u ; \bar{a}_{j} f_{j}(B), U\right\}$. Replace in $U$ every $a_{j} \neq u$ by its representation $f_{j}$ as a $B$-word to get $U=V(B, u)$. Then $D$ is given by $\left\{B_{1}, B_{2}, u ; V\right\}$, showing that the elements $V^{ \pm 1}$ and $\bar{u} f(B)$ generate the same normal subgroup of $F_{3}\left(B_{1}, B_{2}, u\right)$, hence $[1$, p. 157] are conjugates.

$U$ is a function of $a_{h+1}, a_{h+2}$, and $u$, with $a_{h+1}$ and $u$ actually occurring; for certain elements $v_{i}=v_{i}\left(a_{h+1}, a_{h+2}\right) \neq 1$, and integers $x_{i}$,

$$
U=u^{x_{0}} v_{1} u^{x_{1}} v_{2} \cdots v_{k} u^{x_{k}} .
$$

In $G$, the elements $a_{h+1}, a_{h+2}$, and $u$ are connected by the single relation $U$, and so $a_{h+1}$ and $a_{h+2}$ form a free subgroup of $G$ [1, p. 157]. It follows that if $v_{i}\left(a_{h+1}, a_{h+2}\right) \neq 1$, then the representation $w_{i}(B)$ of $v_{i}$ in terms of the free generators $B_{1}$ and $B_{2}$ of $G^{\prime}$ is not the empty word. The representation

$$
V=u^{x_{0}} w_{1} \cdots w_{k} u^{x_{k}}
$$

of $U$ as a $B, u$-word contains therefore the same number of $u$-symbols as $U$ does. On the other hand, viewed as cyclic words, $V^{\epsilon}$ and $\bar{u} f(B)$ are identical, and so both contain $u$ just once. The same must hold for $U=S_{L+h+1}$, hence for $S$; thus, up to conjugation and inversion, $S=\bar{a}_{2} f_{2}\left(a_{0}, a_{1}\right)$. Similarly, $a_{0}$ occurs in $S$ just once, giving

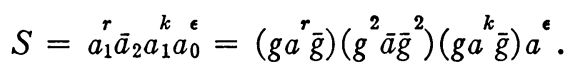

This proves the lemma.

The result above gives $a_{3}=f_{2}\left(a_{1}, a_{2}\right)=f_{3}\left(a_{0}, a_{1}\right)$. The mapping of $a_{i}$ onto $a_{i+1}$ for every integer $i$ is an automorphism $A$ of $G^{\prime}$ given by $A\left(a_{0}\right)=a_{1}, A\left(a_{1}\right)=f_{2}\left(a_{0}, a_{1}\right)=a_{2}$, etc., so that $A^{n}\left(a_{0}\right)=a_{n}=f_{n}\left(a_{0}, a_{1}\right)$; thus the subgroup $K$ of $G^{\prime}$ generated by $a_{0}$ and $a_{1}$ contains every $a_{n}$, is a free group $F_{2}\left(a_{0}, a_{1}\right)$ and contains $G^{\prime}$. Since $K \subseteq G^{\prime}$, it follows that $G^{\prime}=F_{2}\left(a_{0}, a_{1}\right)$. This fact will be needed.

Lemma 2. Let $K=\left\{g, a, b ; g a \bar{g}=a^{x_{1}} b^{y_{1}}, g b \bar{g}=a^{x_{2}} b^{y_{2}}, a b \bar{a}=b\right\} \simeq K^{*}$ $=\left\{g, a, b ; g a \bar{g}=f_{1}(a, b), g b \bar{g}=f_{2}(a, b) a, b \bar{a}=b\right\}$ with

$$
K^{\prime}=K^{* \prime}=F_{2}(a, b) / F_{2}^{\prime}(a, b) ;
$$

let $B$ be the mapping $B(a)=a^{x_{1}} b^{y_{1}}, B(b)=a^{x_{2}} b^{y_{2}}$ in $K^{\prime}$, with matrix $M$, $B^{*}$ the mapping $B^{*}(a)=f_{1}(a, b), B^{*}(b)=f_{2}(a, b)$ in $K^{* \prime}$, with matrix $M^{*}$. Then trace of $M$ equals trace of $M^{* \epsilon}$ and trace of $\bar{M}$ equals trace of $\bar{M}^{* \epsilon} ; \epsilon= \pm 1$.

Proof. Because $K / K^{\prime}=F_{1}(g) \simeq K^{*} / K^{* \prime}$ and the isomorphism $I$ of $K$ onto $K^{*}$ defines an isomorphism $I^{\prime}$ of the factor commutator groups, the image $I(g)$ must be of the form $g^{\epsilon} h(a, b)=g^{\epsilon} h$, and $I\left(K^{\prime}\right)=K^{* \prime}$ with $I(a)=a^{r_{1}} b^{s_{1}}=\alpha, I(b)=a^{r_{2} b^{s_{2}}}=\beta$. 


$$
\begin{aligned}
& B^{*_{\epsilon}}(\alpha)=g^{\epsilon} \alpha \bar{g}^{\epsilon}=g^{\epsilon} h \alpha \bar{h} \bar{g}^{\epsilon}=I(g a \bar{g})=I(B(a))=I\left(a^{x_{1}} b^{y_{1}}\right)=\alpha^{x_{1}} \beta^{\nu_{1}}, \\
& B^{*_{\epsilon}}(\beta)=g^{\epsilon} \bar{g}^{\epsilon}=g^{\epsilon} h \beta \bar{h} \bar{g} \bar{g}^{\epsilon}=I(g b \bar{g})=I(B(b))=I\left(a^{x_{2}} b^{y_{2}}\right)=\alpha^{x_{2} y_{2}}
\end{aligned}
$$

and a similar equality holds for $\bar{B}^{* \epsilon}$.

For any automorphism $X$ and generators $\alpha, \beta$ of $F_{2} / F_{2}^{\prime}, X(a)$ $=N X \bar{N}(\alpha), X(b)=N X \bar{N}(\beta)$, so the equalities above show that the trace is preserved, as claimed.

Under the Nielsen transformation given by $N^{\prime}(a)=a, N^{\prime}(g)=a^{k} g$,

$$
N^{\prime}\left(a_{1}^{r} \bar{a}_{2} a_{1}^{k} a_{0}^{\epsilon}\right)=N^{\prime}(S)=a^{\epsilon} g a^{h} g \bar{a} \bar{g} \bar{g}=W, \quad h=r+k,
$$

up to an inner automorphism (Lemma 1); thus one may assume that $S$ has the form $W$. Since $a \subset G^{\prime}=F_{2}\left(a_{0}, a_{1}\right)$, the sum of its exponents in $S$ is \pm 1 . This gives $h= \pm 1$ for $\epsilon=+1$, and $h=1$ or 3 for $\epsilon=-1$. The word $S$ is thus Nielsen image of one of the following:

$$
S_{1}=a g \bar{a} \bar{g} \bar{g} a g, \quad S_{2}=a g \bar{a} \bar{g} \bar{g} \bar{a} g, \quad S_{3}=\bar{a} \bar{g} \bar{g} \bar{a} g a^{3} g, \quad S_{1}^{*}=\bar{a} g \bar{a} \bar{g} \bar{g} a g .
$$

For $N(a)=a, N(g)=\bar{g}, \bar{N} S_{1}^{*}=\bar{S}_{1}$, so that the first three words remain to investigate. It is now established that a group $G$ of Theorem 4 is Nielsen image of one of the groups $H_{i}=\left\{g, a ; S_{i}\right\}, i: 1,2,3$; it remains to show that these three groups are not isomorphic. This follows from Lemma 2 if they are presented as

$$
\begin{aligned}
& H_{1}^{*}=\{g, a, b ; g a \bar{g}=b, g b \bar{g}=a b\}, \\
& H_{2}^{*}=\{g, a, b ; g a \bar{g}=b, g b \bar{g}=\bar{a} b\}, \\
& H_{3}^{*}=\left\{g, a, b ; g a \bar{g}=b, g b \bar{g}=\bar{a} b^{3}\right\} .
\end{aligned}
$$

To $H_{i}^{*}$ belongs the automorphism $A_{i}$ of $F_{2}(a, b)$ induced by conjugation with $g$ and the matrix $M_{i}$ with trace $c_{i}$ and determinant $\epsilon_{i}$, where

$$
\left(c_{1}, \epsilon_{1}\right)=(-1,-1) ; \quad\left(c_{2}, \epsilon_{2}\right)=(+1,+1) ; \quad\left(c_{3}, \epsilon_{3}\right)=(3,+1) .
$$

This completes the proof of Theorem 4. $\mathrm{H}_{2}$ is the fundamental group of the (trefoil) knots with three crossings, $H_{3}=L$ that of the (Listing's) knot with four crossings. $H_{1}$ does not belong to a knot, since its $L$-polynomial, $-x^{2}-x+1$ [7] is not symmetric in the coefficients [8].

It follows from Theorems 1 and 4 that the group $L$ has non-Nielsen automorphisms but only Nielsen isomorphisms.

Let $A_{i}$ designate automorphisms of $F_{2}(a, b)$.

Corollary 1. If $\left\{g, a, b ; g a \bar{g}=A_{1}(a), g b \bar{g}=A_{1}(b)\right\}=G_{1}$, and $I G_{1}$ $=G_{2}$ is similarly defined, and $G_{1}^{\prime}=F_{2}$, then $G_{2}=N G_{1}$.

Proof. Again the mapping $I$ is of the form $I(g)=g^{e} f_{1}(a, b), I(a)$ 
$=A_{3}(a), I(b)=A_{3}(b)$, and these image words clearly generate $g, a$, and $b$. In particular, if $G_{1}$ is isomorphic to one of the groups $H_{i}$, $i: 1,2$, or 3 , of Theorem 4 , then $G_{1}=N H_{i}^{*}$.

Corol.Lary 2. If the defining relations of

$$
G=\{g, a, b ; g a \bar{g}=A(a), g b \bar{g}=A(b)\}
$$

equal those of $a H_{i}^{*}$ of Theorem 4 taken modulo $[a, b]$, then $N G=H_{1}^{*}$, $N(g)=\bar{w}(a, b) g, N(a)=a, N(b)=b$, for $w \subset F_{2}(a, b)$.

Proof. If $A_{1}(a)=A_{2}(a) \bmod [a, b], A_{1}(b)=A_{2}(b) \bmod [a, b]$, then $A_{1}$ differs from $A_{2}$ by an inner automorphism [5]. Thus the automorphism $A$ of $G^{\prime}$ induced by $g$ differs from the automorphism $A^{*}$ of $H_{i}^{*}$ induced by $g$ by inner automorphisms of $F_{2}(a, b): A^{*}(a)$ $=w A(a) \bar{w}, A^{*}(b)=w A(b) \bar{w}$. Then, under $N$ given above,

$$
\begin{aligned}
N(A(a) g \bar{a} \bar{g}) & =A(a) \bar{w} g \bar{a} \bar{g} w \\
& =\bar{w} A^{*}(a) g \bar{a} \bar{g} w .
\end{aligned}
$$

Similarly for $b$, so that under $N$ the relations of $G$ take the form of the relations of $H_{i}^{*}$, as claimed.

Theorem 5. Let $G=\{g, a, b ; g a \bar{g}=A(a), g b \bar{g}=A(b)\}$ be extension of $F_{2}(a, b)$ by $F_{1}(g), A(a)=a^{x_{1}} b^{y_{1}} \bmod [a, b], A(b)=a^{x_{2}} b^{y_{2}} \bmod [a, b]$, $x_{1}+y_{2}=c, x_{1} y_{2}-x_{2} y_{1}=\epsilon . G^{\prime}=F_{2}(a, b)$ if and only if $(c, \epsilon)=(3,1)$, $(1,1)$, or $( \pm 1,-1)$.

Proof. Let $F=F_{2}(a, b) / F_{2}^{\prime}(a, b), U=a^{u} b^{y}, Y=a^{c} b^{d}, u d-y c= \pm 1$, $N(a)=a^{x} b^{z}, \quad N(b)=a^{p} b^{q}, \quad x q-p z=\epsilon$; set $w=a^{w_{a}} b^{w_{b}} \subset F$. Then

$$
\begin{array}{lll}
(N(U) \bar{U})_{a}=u x+y p-u, & & (N(U) \bar{U})_{b}=u z+y q-y, \\
(N(Y) \bar{Y})_{a}=c x+d p-c, & & (N(Y) \bar{Y})_{b}=c z+d q-d .
\end{array}
$$

The elements $N(U) \bar{U}, N(Y) \bar{Y}$ generate $F$ if and only if

$$
\begin{gathered}
\pm 1=(u x+y p-u)(c z+d q-d)-(u z+y q-y)(c x+d p-c) \\
\quad=(u d-y c)((x q-p z)+1-(x+q))= \pm(\epsilon+1-(x+q)),
\end{gathered}
$$

that is if the pair of values $(x+q, \epsilon)$ is either $(3,1)$, or $(1,1)$, or $( \pm 1,-1)$. The proof is based on this fact.

If the condition on $(c, \epsilon)$ holds then $g a \bar{g} \bar{a}=A(a) \bar{a}$ and $g b \bar{g} \bar{b}=A(b) \bar{b}$ generate $F$, so that together with conjugates of $a b \bar{a} \bar{b}$ they generate $F_{2}(a, b)$. Then $F_{2}(a, b) \subset G^{\prime}$; but since $G^{\prime} \subset F_{2}(a, b) \subset G, F_{2}(a, b)=G^{\prime}$, as claimed.

If $F_{2}(a, b)=G^{\prime}$ then it is generated by $g a \bar{g} \bar{a}, g b \bar{g} \bar{b}, a b \bar{a} \bar{b}$ and their conjugates in $G$, and $G^{\prime} / G^{\prime \prime}$ is generated by $g a \bar{g} \bar{a}=\alpha, g b \bar{g} \bar{b}=\beta$ and 
their conjugates by $g^{k}, k: \pm 1, \cdots$. If $g a \bar{g}=a^{x} b^{y}, g b \bar{g}=a^{u} b^{v}$ modulo $G^{\prime \prime}$, they generate $G^{\prime} / G^{\prime \prime}$, and $\alpha=a^{x-1} b^{y}, \beta=a^{u} b^{v-1}$ in $G^{\prime} / G^{\prime \prime}$. As $g \alpha \bar{g}=\left(a^{x} b^{y}\right)^{x-1}\left(a^{u} b^{v}\right)^{y}=\left(a^{x-1} b^{y}\right)^{x}\left(a^{u} b^{v-1}\right)^{y}=\alpha^{x} \beta^{y}$, and similarly $g \beta \bar{g}$ $=\alpha^{u} \beta^{v}$, the elements $\alpha, \beta$ already generate the factor group $G^{\prime} / G^{\prime \prime}$. As $\alpha=A(a) \bar{a}, \beta=A(b) \bar{b}$, the condition on $(c, \epsilon)$ must hold, as claimed.

It may be noted that for $\epsilon=-1$, the inverse transformation $\bar{A}$ has the trace $-c$, so that only three possibilities remain modulo the Nielsen transformation $N(a)=a, N(b)=b, N(g)=\bar{g}$.

TheOREM 6. The group $G$ of Theorem 5 is isomorphic to one of the groups $H_{i}^{*}$ of Theorem 4 if and only if $(c, \epsilon)=\left(c_{i}, \epsilon_{i}\right)$.

Proof. The necessity of the condition follows by an easy step from Lemma 2. Suppose the condition satisfied. Designate by $(A)$ the group of all automorphisms of $F_{2}$, by $(I)$ the group of inner automorphisms of $F_{2}$, and by $\left(A^{\prime}\right)$ the group of automorphisms of $F_{2} / F_{2}^{\prime}$; then $\left(A^{\prime}\right)$ is given by the modular group and $\left(A^{\prime}\right) \simeq(A) /(I)$ [5]. It is known [9] that in the domain of unimodular integral two-by-two matrices (the modular group) the class number for trace $c_{i}$ and determinant $\epsilon_{i}, i: 1,2,3$, is one; hence the matrix $M$ belonging to $G$ (see Lemma 2) is a conjugate $N M_{i} \bar{N}$ of the matrix $M_{i}$ belonging to $H_{i}^{*}$, with $|N| \pm 1$. This, together with $\left(A^{\prime}\right) \simeq(A) /(I)$, gives: $A=N A_{i} \bar{N}$ (see Lemma 2), and so $G=N H_{i}^{*}$, as claimed.

\section{BIBLIOGRAPHY}

1. W. Magnus, Ueber diskontinuierliche Gruppen mit einer definierenden Relation (der Freiheitssatz), J. Reine Angew. Math. vol. 163 (1930) pp. 141-165.

2. - Untersuchungen ueber einige unendliche diskontinuierliche Gruppen, Math. Ann. vol. 105 (1931) pp. 52-74.

3. A. Kurosh, Group theory, Chelsea, 1956, 308 pp.

4. B. H. Neumann, Some remarks on infinite groups, J. London Math. Soc. vol. 12 (1937) pp. $120-127$.

5. J. Nielsen, Isomorphie der allgemeinen unendlichen Gruppe mit zwei Erzeugenden, Math. Ann. vol. 78 (1917) p. 392.

6. M. Dehn, Die beiden Kleeblattschlingen, Math. Ann. vol. 75 (1914) pp. 402-413.

7. R. H. Fox, Free differential calculus, II, Ann. of Math. vol. 59 (1954) p. 204.

8. K. Reidemeister, Knotentheorie, Chelsea, 1948, p. 40.

9. O. Taussky, On a theorem of Latimer and MacDuffee, Canad. J. Math. vol. 1 (1949) pp. 300-302.

STOCKBRIDGE, MasS. 